\title{
Téoros
}

Revue de recherche en tourisme

\section{Un regard critique sur la mise en tourisme de l'exotisme tribal dans le Nord thaïlandais}

\section{Jean Michaud}

Volume 13, numéro 2, été 1994

Regards sur le tourisme dans les pays en développement

URI : https://id.erudit.org/iderudit/1077762ar

DOI : https://doi.org/10.7202/1077762ar

Aller au sommaire du numéro

Éditeur(s)

Université du Québec à Montréal

ISSN

0712-8657 (imprimé)

1923-2705 (numérique)

Découvrir la revue

Citer cet article

Michaud, J. (1994). Un regard critique sur la mise en tourisme de l'exotisme tribal dans le Nord thaïlandais. Téoros, 13(2), 34-38.

https://doi.org/10.7202/1077762ar d'utilisation que vous pouvez consulter en ligne. 


\title{
Un regard critique sur la mise en tourisme de l'exotisme tribal dans le Nord thailandais
}

\author{
Jean Michaud"
}

La Thailande est certes l'un des plus intéressants exemples de développement par l'industrie touristique des trente dernières années. Située dans ce qu'il était convenu d'appeler jusqu'à récemment le Tiers Monde et qu'on désigne plus volontiers aujourd'hui, suivant les conceptions économiques et politiques auxquelles on se réfère, comme le Sud, la Périphérie, les Pays moins développés, ou les Nouveaux pays industrialesés, la Thailande partage avec quelques autres destinations - quon pense au Kenya et aux Philippines - une réputation du succès sur le plan financier associé à une industrie touristique du sexe florissante. De nombreux écrits traitent de cette association douteuse qui a fini, avec les années, par résumer dansl'esprit d'une majorité de visiteurs étrangers ce qu'a la Thaillande à offrir au touristeoccidental. Leschiffresde l'affluence touristique en constante croissance depuis 1960 viennent confirmer l'efficacité d'une formule décriée par de nombreux observateurs, et recherchée par d'autres plus nombreux encore. Pour éviter de se complaireà rouler dans ces mêmes ornières de la recherche touristique en Thailande, creuses déja du passagerégulier de très nombreux auteurs - surtout occidentaux -, c'est sur un autre aspect du tourismeen Thailande qu'il est proposé de porter ici le regard.

Dans les trois cas cités plus haut, et dans d'autres encore, une attraction occupe une place de choix dans la publicité faite à l'endroit des visiteurs étrangers, une place hors de proportion avec la faible importance démographique des populations faisantl'objet de cette promotion. Il s'agit des populations qu'on dit tribales. Exotisme fortement teinté de primitivisme, traditions vestimentaires distinctes, vivantes et colorées, isolement dans les lieux reculés des périphéries nationales garant d'authenticité culturelle, toutes ces caractéristiques qui servent dans la publicité touristique à décrire ces minorités sud-est

Monsieur Jean Michaud est anthropologue, chercheur associé au Groupe d'études et de recherche sur l'Asie contemporaine de l'Universite Laval à Québec.

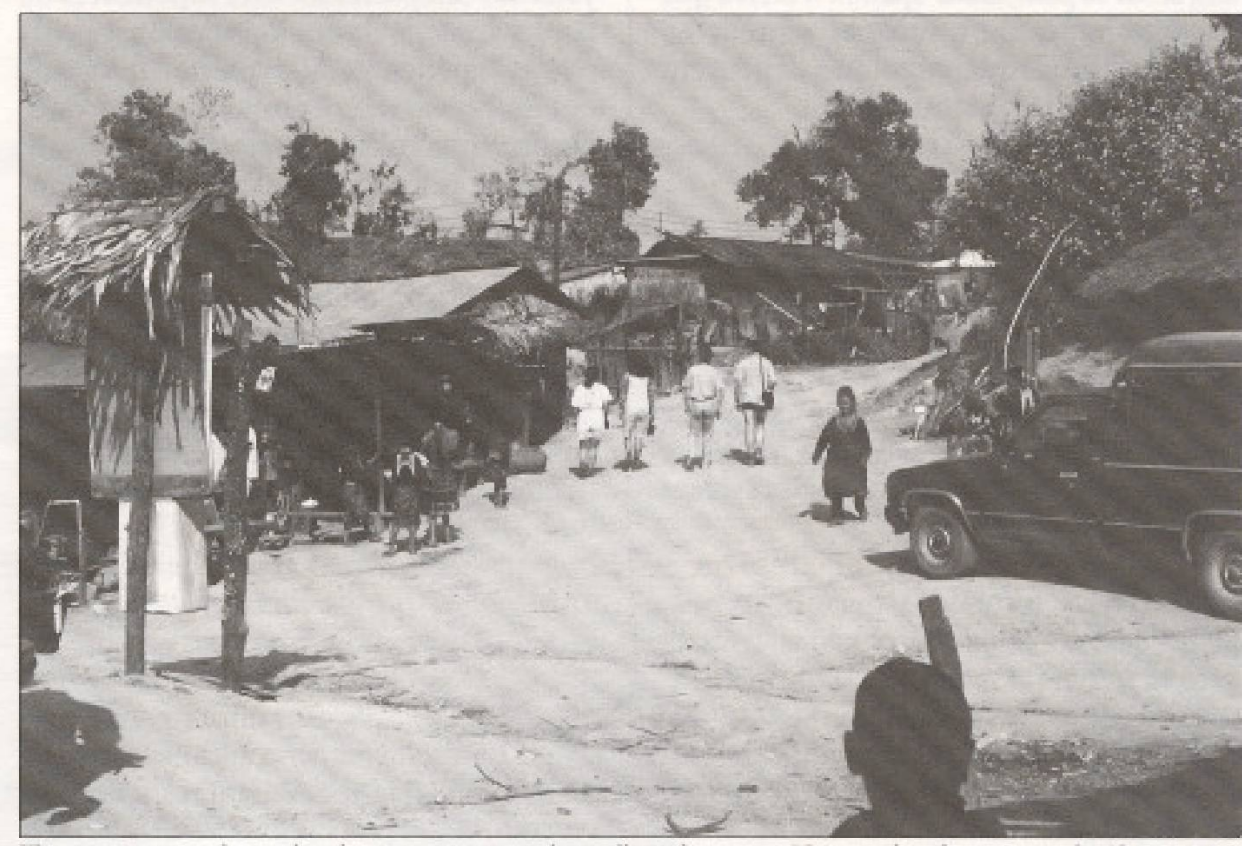

Touristes quittant leur vehicule pour visiter à pied un village du groupe Hmong dans ha provinee de Chiang-Mai.

asiatiques sont aussi convenues que celles utilisant ici l'igloo et les peaux de phoque pour résumer la spécificité inuit.

Ces populations tribaler sont ainsi mises en scène parce qu'elles incarnent l'exotisme. Et en Occident, l'exotique vend, très bien même. Mais qu'en est-il sur le terrain? Comments'articule sur placecette miseen marché et qui en sont les gagnants? Parmi les 12 millions d'habitants du Nord = sur les 57 millions d'individus que compte la Thailande -, moins de 600000 montagnards véritables, peu lettrés et mal aguerris à l'économie de marché peuvent-ils rivaliser avec les populations thai des basses-terres dans ce qui est devenu une activité hautement compétitive exigeant des compétences particulières? Il est proposé ici d'examiner la situation pour tenter, en fin d'analyse, de comprendre les conséquences de la promotion de l'exotique et du tourisme d'aventure dans le Nord de la Thailande.

\section{Quelques chiffres sur le tourisme pour le pays et sa partie Nord}

En 1990, nous apprend le rapport annuel du Tourism Authority of Thailand
(TAT, 1991), 5298860 visiteurs internationaux identifiés comme touristes (définition, Ibid.: 4) sont entrés au pays, une augmentation de $10,17 \%$ sur l'année précédente et de $6414 \%$ sur $1960(81340$ personnes pour cette première année de statistiques touristiques). En particulier, l'année 1987, exhaussée grâce au succès promotionnel d'une Visit Thailand Year, a causé à elle seule une augmentation de $23,59 \%$ sur l'année précédente, et fut suivie d'une augmentation de $21,47 \%$ l'année suivante. De 1960 à 1990 , les revenus bruts du tourisme international pour l'ensemble du pays sont passées de 196 millions à 111 milliards de baht (ou de 7,84 millions à 4,42 milliards SUS, 1 SUS = environ $25 \mathrm{baht}$ ), une augmentation de $56414 \%$ alors que depuis l'année 1989 , l'augmentation s'élève à 14,7\%. Activité particulièrement lucrative, l'excédent des revenus du tourisme international sur les dépenses pout l'année 1990 se chiffre à 88,8 milliards de baht (ou 3,47 milliards SUS).

En comparaison, les revenus bruts en devises étrangères du tourisme surpassent de 26,1 milliards de baht, ou $31 \%$, ceux du premier secteur de l'exportation 
thailandaise: les produits textiles (ces deux sources de devises étaient nez-à-nez en 1985), et de 71,9 milliards de baht, ou 186 $\%$, ceux du second secteur en importance: lesordinateurs et pièces d'ordinateurs. $\mathrm{Ou}_{1}$ pour faire image en cette Asie rizicole, les revenus du tourisme pour l'ensemble du pays équivalent à peu près à quatre foisceux de la première exportation agricole du pays: le riz (premier rang mondial en 1992, troisième secteur national d'exportation) (TAT, 1991: 45). C'est donc dire que l'apport économique lié à l'industrie touristique constitue une source de revenus majeure pour l'État thaillandais, et procure un effet relaxant à une balance commerciale déficitaire ( 8 milliards SUS en 1992) et une dette extérieure considérable $(35,8$ milliards SUS en 1991) (État du monde, 1993: 350-351).

Il est difficile desavoir quelleest exactement la part rejoignant la région du Norddans ce flux des personnes et des devises. Un fractionnement des données nationales par région (TAT, non daté) fondé sur une méthodologie que l'on n'a pas expliquée dans le rapport, propose pour 1990 le nombre de 592780 visiteurs étrangers dans la province de Chiang Maï, contre 2 190891 visiteursde nationalité thailandaise. L'office du TAT de Chiang Maï fournit quant à lui un nombre de 562000 touristes étrangersayantvisitéla provincedeChiang Maï en 1990, un chiffre indiqué comme étant approximatif et dont la méthode de collecte est également gardée secrète. Par ailleurs, revenant au rapportannuel national duTAT (1991), il est possibled'arriver, par un calcul trop long pour être détaillé ici, à une estimation de 1558074 touristes ayant résidé à Chiang Maï ou dans sa province. Ce nombre, nettement plusélevé que ceux fournis par les différents organes du TAT, aurait l'avantage de prendre en compte les nuits passées dans des établissements d'hébergement négligeant la procédure d'enregistrement, ou encore passées dans les transports en commun, chez des particuliers, ou simplement en trekking, cedont les registres hôteliers ne gardent pas la trace.

Quoi qu'il en soit, sur le total de visiteurs dans la province de Chiang Maï, le bureau de Chiang Maï du TAT propose pour 1990 une approximation du nombre de visiteurs s'adonnant au trekking dans les montagnes de la région, soit 62210 . Encore une fois, la méthodologie utilisćen'est pas explicitée. Il est prudent de considérer ce nombre officiel comme assez conservateư et pouvant vraisemblablement être augmenté si l'on voulait obtenir un estimé plus réaliste de la circulation touristique étrangère dans les montagnes du Nord.

\section{L'État et le développement du tourisme}

Tourism planning reflects wider realities of power in the saciety. If the government is authoritarian and structures of democratic input are lacking, if a small political elite is allied with comprador and foreign capital, if the ideology of development is capitalist, there are fow grounds for expecting Tourism planning and development to bave different social consequences than otber *developmentmpolicies. (Wood, 1984:363)

De manière générale, quand elle se déploie entre Centre et Périphérie, la forme privilégiée qu'adopte l'industrie touristique transnationale ne differe pas de celle que prend n'importe quelle autre industrie de services capitaliste (Britton, 1982; Hoivik and Heidberg, 1980; Lanfant, 1980; Nash, 1989).

Les acteurs directement impliqués dans le tourisme international(1) se répartissent entre deux pôles: la source, soit un pays (région, zone, etc.), du Centre et sa clientèle touristique, et la destination, soit un pays (région, zone, etce), de la Périphérie qui dispose de l'attraction à visiter. Les échanges sont fondés sur des relations inégalitaires modelées sur des rapports économiques déjà en place entre les deux partenaires.

Dans son étude des politiques touristiques en Asie, Linda Richter (1989; voir également 1993) observe quele laisser-faire a été l'attitude adoptée par les dirigeants politiques à l'égard de l'implantation et du développement d'une industrie touristique sur le territoire thailandais, qu'elle soit d'origine nationale ou commandée de l'extérieur. Débutée en force avec l'adoption de Bangkok, au début des années 1960, comme lieu privilégié pour les périodes de R \& R (Rest and Recreation), récompensant les militaires américains engagés en Indochine, un accroissement régulier de la clientèle non-militaire à la fin des années 1960 et au début des années 1970 a contribué à tempérer le manque à gagner qui n'a pas manqué de survenir après le départ définitif des troupes en 1975. Depuis, l'industriedu tourismeen Thailande fonde deplus en plusses activités sur une dientèle internationale de groupes organisés et de gens d'affaires. Mais les plus grands fournisseurs de clientèle touristique demeurent lesvoisins régionaux, avecen têtela Malaisie et les pays aisés d'Asie de l'Est; chez ces clientèles, il est notoire que la consommation de produits sexuels est un incitatif important, comme l'indique la plus forte proportion de mâles voyageant seuls en comparaison des autres origines nationales.

En fait, jusqu'aux années 1980, les gouvernants ont résisté à l'idée d'une implication active de l'État dans la régulation, le soutien et la promotion du tourisme au pays. La libre-entreprise pouvait ainsi s'activer dans les meilleures conditions, au moins pour celles disposant des meilleurs appuis tant dans l'appareil étatique que dans la classe politique proprement dite. Une baisse du flux touristique en 1983, une montée de la criminalité dans les stations balnćaires telle Pattaya et une réputation de plus en plus tenace de marché ouvert du sexe, que l'État a été très lent à reconnaître officiellement quoiqu'il en dirigeât les destinéesen coulisses, ainsi qu'un symbole, soit le fait que les revenus du tourisme en devises étrangères dépassent ceux du riz vers 1982 , ont incité l'Etat à l'action. Alors que le troisième Plan de développement national (1972-1976) avait intégré le tourisme comme une composante - nominale - de la planification gouvernementale, c'est au cinquième Plan (1982-1986) que l'État reconnaît pour la première fois le développement touristique comme une priorité économique majeure, sans toutefois que cette formalisation s'accompagne de l'emphase politique qui devrait lui ểtre associée (Richter, 1989;86). Richter note qu'à la fin de la décennie 1980, athe government's evaluation of the tourist sector continues to be almost exclusively in economic terms with little attention given to the distribution of income and the sociocultural impact of the rapid development of the tourist industry (1989: 87), alors qu'un objectif explicite du TAT en 1983 expose une intention louable: *Conserving natural resources and environment and reviving cultureinorder to retain Thai identityatits best (TAT, 1983: 15).

Concernant le tourisme dans les populations montagnardes, l'une des principales attractions du Nord, la position gouverne- 
mentale est mitigée. L'État aime à présenter une image progressiste du pays dans son ensemble et récuse une réputation de producteur d'opium et d'héroine. Mais il s'avère que *the TAT chooses to highlight "simple, unspoiled" tribal life and often shows pictures of tribal groupsoutworking in their colorful poppy fields» (Richter, 1989: 96; voir également Cohen, 1989). Si cet exemple illustre bien lesécarts de points de vue promotionnels et l'absence d'une politique explicite au sein même de l'appareil étatique, la ligne directrice de la politique touristique à l'égard des montagnards est en fait assimilable à celle s'appliquant à l'ensemble de la population, et aucun objectif de développement touristque n'est explicitement accolé à ces populations périphériques. Lacitation terminant le paragraphe précédent illustre cette situation.

\section{Les discours promotionnels, le must tribal et la clientèle}

Qu'il s'agisse d'encarts publicitaires sur les murs des agences de voyages et dans les magazines, des photos accrocheuses dans de nombreux coffee table books, des pages couvertures de guides écrits et de cartes géographiques grand public, l'image promotionnelle comme le discours sur la Thailande et les Thailandais offerts à la consommation touristique se résument à quelques clichés: l'architecture religieuseincluant démons et Bouddhas variés -, l'activité balnéairetropicaleet ses attributs, la tradition exotiquecoupée de modernisme urbain, l'éternel sourire et, enrobédetoutes les périphrases qui se puissent imaginer, l'espoir de la rencontre amoureuse pour la durée des vacances ou pour la vie. Baffie (1988) a effectué une éloquente revue du contenu des guides touristiques sur la Thailande écrits en langue française, plus de 30 sans compter les rééditions, et a mis en évidence la reprise systématique des clichés consacrés charpentantcemonument difforme.

Mais également, et bien au-delà de l'importance démographique des populations auxquelles elle se réfere explicitement, l'imagerie tribaliste est omniprésente, le plus souvent classée sous la rubrique de l'exotisme et de l'aventure. Il n'y a pas d'ouvrage luxueux ni de guide touristique écrit sur la Thailande qui ne comporte son chapitre tribal, d'abord pour en magnifier l'originalité culturelle, le bon état de conservation des traditions (surtout dans leurs manifestations visibles), la simplicité accueillante, suivi de quelques phrases pour souligner aujourd'hui la déplorable entrée du modernisme et de la gadgetrie dans ce monde social jusquelà gratifiéd'une pureté virginale.

Pour les Thaillandais de cultura taï, soit l'immense majorité des habitants du pays, les montagnards de culture non-taî sont perçus comme des agriculteurs attardés, illettrés, inefficaces dans leur activité agricole, voire carrément nuisibles à l'environnement comme à la sécurité de l'État (Arbhabhirama et al., 1988), et le discours officiel de l'État n'est pas sans être une cause de cette perception faussée. Baffie (1989) a résumé cet état d'esprit au moyen d'une intéressante analyse de la représentation des montagnards et de leurs cultures dans les bandes dessinées à deux sous dont sont friands les Thailandais. Pour cette raison, donc, mais aussi à cause d'une vision du monde et d'uneconception du loisir qui ne valorise pas la connaissance, la découverte et l'aventure, il est donc compréhensible que la visite touristique de villages montagnards à l'intérieur même des frontières nationales ne soit pas une activité désirable dans l'imaginaire collectif des nationaux.

La clientèle touristique que l'on retrouve dans le Nord n'est pas exactement représentative de l'ensemble des visiteurs entrant au pays. Elle répond à certaines caractéristiques. Paraphrasant Cohen, Richter avance qu'en dépit de l'expansion del'industrie du sexe pour étrangers jusque dans les villes secondaires telles Chiang Maï ou Hat Yaĩ, constituant en soi une cause de tourisme hors de la métropole, sthose who go to the North of Thailand are moreapt to be interested in the culture, the tribal groups, and interaction with the Thais»(1989:96). Bien que fondée sur des impressions plus que sur des évaluations statistiques, cette affirmation a toutes les chances d'être véridique, du moins concernant l'intérêt à l'égard des groupes montagnards. C'esteffectivement au Nord que setrouvent ces sociétés, loinde Bangkok et ses attraits, et pl us loin encore des stations balnéaires. Si toutefois il est juste de prétendre que cette ouverture va jusqu'à un intérêt réel pour la culture ou un désir profond d'entrer en relation avec des Thaiis, cela reste à prouver. $\mathrm{A}$ contrario, il est certainement permis de penser que la motivation de ces visiteurs du Nord n'est pas uniquement tributaire des facilités du modernisme industriel urbain, ni de la compulsion du plagisme. Du moins pour cette partie de leur itinéraire.

Suivant mon observation, non-systématique, la clientèle touristique étrangère du Nord paraît se diviser - à peu près également, dirais-je - entre deux grandes catégories. Il y a les groupes organisés de courte durée, les package deal, pour lesquels le Nord est une étape dans un grand tour organisé du pays, logeanten hôtels, souvent des chaînes internationales tels Novotel ou Holiday Inn, intéressés à l'atmosphère typique ou autbentique de cette région périphérique, à son climat plus frais, et à une certaine proximité avec la nature, ce qui inclut des visites de champs de pavot autorisés pour cet effet, de camps d'entraînement d'éléphants, de fermes de papillons, d'orchidées et de serpents. Une fois sur place, la formule privilégiée est le dicytrip en autobus nolisé, et la sortie comprend généralement la visite d'un village tribal, clou de la journée: pendant une heure ou deux, on aime $y$ acheter des souvenirs, on observe ces primitifs colorés, on prend des photos, maison communique très peu en raison de l'écart linguistique. L'autre grandecatégorie est disparate dans sa forme, mais plutốt uniforme quant à ses activités privilégiées. Ce sont des voyageurs autonomes ou en petits groupes, moins pressés par le temps, à budget plus restreint - ce qui ne présume pas que ces voyageurs ne disposent pas des moyens financiers pour un séjour plus luxueux - et à la recherche d'émotions fortes, uniques, d'expériences humaines marquantes. Celles-ci prennent diverses formes suivant le degré de sensibilisation à la culture locale. Les moins informés recherchent l'excitation dansl'inconnu(exploration autonome du territoire, fuite des lieux du tourisme de masse, nuits d'ivresse, essai des drogues locales), et adopteront la même structure comportementale dans les autres pays où les mènera leur voyage, reflétant par là les généralisations au moyen desquelles leur esprit conçoit les ensembles culturels qu'ils côtoient. Leur horaire est souple, l'impulsion du moment tient lieu d'agenda. Les plus préparés à la culture locale quant à eux cherchent à s'extraire de leur cadre culturel habituel, recherchent les contacts avec les habitants du pays et aiment à apprendre toujours plus sur les moeurs locales, préférablement au moyen d'expériences personnalisées. Ils tendent à avoir un horaire plus construit et ils s'imposent volontiers des tâches à accomplir, des ob- 


\section{Thaïlande : Provinces et régions}

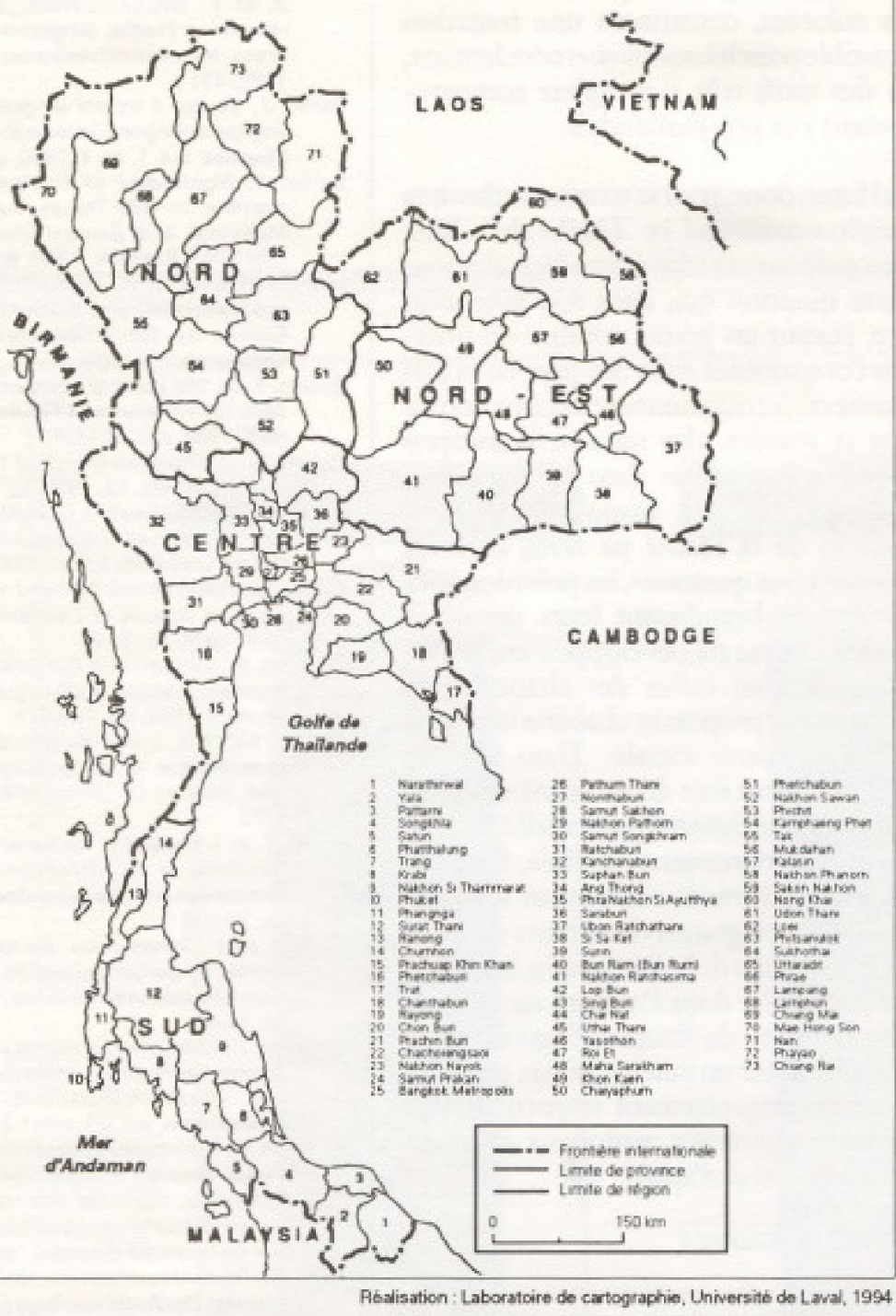

jectifs à atteindre durant le séjour. Eưre personnellement au contrôle de toutes les dimensions du voyage est pour eux une valeur cardinale. Dans tous les cas de cette seconde catégorie, une formule privilégiée est le trekking - une activité aux frontières fluctuantes comprenant généralement une part d'activité physique, une part de confrontation à la Nature, et une part de rencontre avec l'Autre exotique. C'est au moyen de celui-ci que le voyageur entrera en contact avec les populations montagnardes vivant en villages isolés.

Pour situer ce genre de visiteurs dans l'éventail des motivations pouvant animer les touristes en général, la typologie mise de l'avant par Valene Smith (1989) peut être utiliséeavecprofit. Cettetypologie fut proposée dans la premièreédition de Hosts and Guests en 1977. Elle comprend 5 types: ethnic Tourism, cultural Tourism, bistorical Tourism, environmental Tourism et recreational Tourism. Trèssynthétique, elle est utile pour classifier de manière générale les motivations les plus courantes des touristes, surtout ceux issus de sociétés industrialisées. Elle s'avère en outre, à mon avis, moins rigide quelescatégories fondées sur lecomportement, proposées par Cohen $(1979)^{(0)}$. Suivant cette typologie donc, nous aurions affaire ici à un tourisme ethnique (ethnic Tourism) et à sa correspondance habituelle, le tourisme environnemental (envirummental Tourism). Ethnique parce que son adepte recherche des particularités ethnologiques exotiques qui distinguent ces visités des autres et les rendent uniques à ses yeux; environnemental parce que l'écosystème visité ressort du commun - toujours dans l'oeil de l'observateur étranger -, qu'il mérite en soi d'être visité, et qu'il permet des activités exigeantes auxquelles tous ne peuvent prétendre. Cette clientèle est généralement jeune, relativement sćrieuse, capable et désireuse de se mériter par un certain effort ce qu'elle rapportera de souvenirs et d'émotions.

Ce qui ne veut pas dire que le nombre absolu de touristes recherchant ce type de voyage n'est pas important. Au contraire, on constate depuis une vingtaine d'années que la demande pour le tourisme ethnique et environnemental - que l'on qualifie généralement dans l'industrie de tourisme d'aventure - est constamment à la hausse ${ }^{(4)}$, surtout lorsque comme depuis quelques années, les instigateurs savent relever leur discours promotionnel par un savant dosage de valeurs humanistes en vogue.

\section{De quelques conséquences}

J'ai décrit ailleurs, en mettant le cas thailandais en rapport avec un cas comparable au Kashmir Indien, quels sont les avantages économiques et politiques que peut tirer l'État à laisser faire, et même favoriser l'expansion du tourisme ethnique et environnemental dans les périphéries nationales sensibles sur le plan góostratégique et occupées par des minorités peu intégrées à l'identité nationale (Michaud, 1993). En résumé, le pouvoir central peut trouver dans ce type d'activité économique un catalyseur efficace de l'acculturation des minorités au groupe dominant, ici les Thaï, et faire l'économie par la mème occasion d'un bon nombre d'investissements coûteux par rapport à la faible démographie dans ces régionsisolées. Une tentation à laquelle l'Etat ne peut résister.

De plus, le tourisme d'aventure en région périphérique permet de faire tourner tout un pan de l'économie dans les capitales régionales, ici Chiang Maï, en particulier dans le secteur des services et de la petite entreprise, des secteurs incertains et fluctuants. Le nombre d'agences de trekking à Chiang Maï de même que le nombre de personnes engagées par cellesci reste un mystère, mais ces deux chiffres ne sauraient être que considérables. À Chiang Maï, en plus du circuit des forfaits 
tout compris, toute la dispensation des services complémentaires aux touristes d'aventure est entre les mains d'intermédiaires thais, qu'ils soient indépendants ou qu'on les ait mandatés depuis Bangkok; ce sont ces sous- et sous-sous-traitants dont il a été fait mention plus haut. Ce qui est certes fort bien du point de vue de l'appropriation locale de ce segment d'un produit qui trouve son origine à l'étranger et tombe sous le contrôle de l'industrie touristique internationale. De l'accueil de la clientèle à son guidage dans les montagnes en passant par l'hébergement, la sustentation, le transport et les divertissements, des Thai se posenten intermédiaires et prélèvent chacun une part du bénéfice mis en disponibilité par le voyageur (sans le chiffrer, Cohen (1983) a également fait ce constat).

Mais qu'en est-il du partage de l'assiette pour ces autres partenaires, ces minorités exotiques non-tai qu'on est venu visiter de si loin? Dans les villages de montagnes, ce sont les intermédiaires extérieurs qui empochent leplus gros des bénéfices. Suivant mon calcul, fondé sur l'examen approfondi d'un cas représentatif et sur l'observation complémentaire de quelques villages montagnards que visitent les trekkers, la part de l'investissement du trekker pour l'ensemble de son trek (sans même prendre en compte toutes les dépenses du voyage qui ne sont pas directement reliées à cette activité spécifique), la part, donc, qui atteint effectivementles montagnards cux-mêmes est de $3 \%$ seulement (Michaud, 1994). Le reste, $97 \%$, est pour les intermédiaires. Le tourisme d'aventure dans le Nord thailandais est une activité économique profitable certes, mais certainement pas pour ces gens qui sont au coeur du produit touristique. Pourtant, l'acculturation massiveet rapide, la modernisation et, dans bon nombre de cas, la paupérisation de ces montagnards dont l'originalité nourrit la stratégie de promotion touristique pour toute cette région du Nord thailandais et, dans une bonne mesure, pour tout le reste du pays, constituent un danger pour l'industrie touristique locale, une sorte de bombe à retardement dont l'implosion risque d'emporter avec elle la poule aux oeufś d'or. Jusqu'à maintenant, nil'État, ni l'industrie ne manifestent l'intention de réfléchir sur la menace pour l'économie locale que constitue l'érosion rapide de l'authenticité de la resource. Non plus qu'une réflexion plus large sur le tourisme tribal, ou d'aventure, ne semble être en cours.
N'oublions pas que sur le plan régional, l'ouverture de plus en plus grande du Vietnam et, bientôt, du Laos et du Yunnan chinois, tous largement pourvus en minorités colorées, constituera une tentation irrésistible pour les aventuriersoccidentaux, et à des tarifs très avantageux comparativement aux prix thailandais.

Quel futur, donc, pour le tourismeethnique et environnemental en Thailande? Bien malin qui pourrait répondreadéquatement à cette question qui, dans son acception large, rejoint un grand nombre de situations comparables dans des pays de la Périphérie où se maintiennent encore, contre vents et marées, des minorités exotiques mises à contribution dans le marketing touristique national. Jusqu'à ce que l'observation de la réalité ne nous livre les réponses à nos questions, les prévisions des économistes brandissant leurs dernières grilles d'analyse du développement régional valent bien celles des chapelles du management proposant chacune leur stratégie d'ingénierie sociale. Dans tous les cas, il ne saurait être décemment soutenable que dans la dynamique actuelle, où une industrie extrêmement ramifiée, fluide et compétitive affronte ou, suivant ses intérêtts, s'allie aux grands détenteurs de capitauxet aux États de la Périphérie, le paysan illettré et isolé, dont l'univers ne dépasse guère la limite du finage villageois, ait la chance de jouer un autre rôle que celui qui lui est péremptoirement imparti par les décideurs régionaux, nationaux et internationaux: celui d'appât. $f$

\section{NOTES ET RÉFÉRENCES}

(1) Dans le cas du tourisme de masse, par exemple. ou encore concernant le tourisme entre pays du Centre, bien des acteurs supplémentaires d ceux. presentes icientrent dans oe jeu. Pour un portrait plus complet décrvant les rapports Centre-Péri. phérie, on se référera utilement de la premère partie de l'article the Structure of International Tourisn and Tourism development in Kenya, de Thea Sinclair et al (1992), et au Tourisme entre le Centre et la Peripherie et autodependance, de Hoivik theidberg (19e0). Pour les besoins de la démenstration, nous comiendrons que la trame simple que je propose ici schematise comenablement la structure de l'industrie touris. tique internationale du package deal associé au tourisme ethnique et environnemental, celui qui, on leverra plus loin, fournit enclientélele tourisme d'aventure du Nord.

(3) Mais elle gagnerait a titie précisde quant a la différence entre tourisme culturel et tourisme ethrique, deux types qui, sur le terrain, sont difficiles a distinguer. Robert Wood a proposé une alternative a ce dilemme (1984: 360-362).

(4) Au Club Aventure, une agence québécoise crébe en 1973 etspecia lisee dans les forfaits de groupes avec guides vers des destinations aventureuses, on note un accroissement régulier des ventes depuis l'ouverture, avec, strangement, les havs ses les plus importantes durant les périodes de déficience de l'économie au Centre lcommunication personnelle, hiver 1994).

Arbhabhirama, A. PHANTUMVANIT, D, ELKINGTON, J. et P. INGKASUWAN. Thailand, natural resources Profile, Singapour, Oxford University Press, série natural resources of South-East Asia, $198 \mathrm{~B}, 431 \mathrm{p}$

Baffie, J., Voyage a travers les guides. Les guides de voygge de langue francaise sur la Thailande, Inter. Mondss, vel, 1, no 1, 1968, po 103-164.

Baffie, J., Highianders as Portrayed in Thai Penmy Horrible, in Hill Tribes Today, ed. by John Mckinnon and Bernard Vienne, White LotusORSTOM, Bangkok, 1989, pp. 393-408.

Bhangananda, K et W. WIWATSORN, Manuel pour guides touristlques (edition françaisel. Bangkok. Centre de formation continue. Universite Chulalongkorn, 1989.

Britton. S.G., the political Economy of Tourism in the Third World, Annals of Touriam Research, wol. 9. no 3. 1982, pp. $331-358$

Cohen, E., A Phenomenology of tourist Experiences, Sociology, vol. 13, 1979, pp. 179-201.

Cohen, E. Hin Tribe Tourism, in Highlanders of Thailand, in J. MeKinnonet W. Bruksasri (eds), Kuala Lumpur, Oxford University Press, 1983, pp. 307-325.

Cohen, E. Primitive and Pemote: Hill Tribe Trekking in Thailand, Annals of Tourlsm Research, vol, 16, no 1,1599 , pp. 30-61

Dearden. P. Tourism and Sustaingble develooment in Northern Thailand, the Geographieal Revlew wol. 18, no 9, 1991, PP. 400-413.

État DU MONDE, L'stat du monde 1994. Annuaire deconomique t geopolitique mondial, Montréal, Editions La DesouvertelÉditions du Boréal, 1993.

Hoivik. T. et T. Heidberg. Tourisme entre le centre er k peripherie et outodependance, Revue intornationale des sciences sociales, vol, 32, no 1, 1980 , op. $74-108$.

Laniant, M.F. Introduction Le tourisme dans le pro. cessus dinfernationalisation, Revus Internationale des sciences soclales, vol. 32, no 1, 1980, pp. $14-45$

Michaud, d. Catalyst of economic and political Change. Tourism in Highland Minorities in Ladakh (India) and Northern Thailand, Internationale Asienforum, vol, 24, nos 1-2, 1993, pp. 21-43.

Michaud, J., Ressistance ot flexibilité. Le changement social dans un village Hmong du Nord de la Thailande, these de doctorat en anthropologie sociale. Département d'anthropologie de l'Université de Montréal (Canada). 1994

Nash, D. Tounimas a formofimcerialism, in Hosts and Guests: the Anthropology of Tourism. Second Edition, ed, by Valene $H$. Smith, University of Pennsylvania Press, Philadelphia, 1989, pp. 37-52

Fichter, L.K., the Politics of Tourism in Asia, Honolulu. University of Hawail Press, 1989.

Richter, L.K. Tounism Policy-Making in South-East Asis, in M. Hitchcock V.T. King, M.J.G. Parnwell (eds). Tourim in South-East Asia, Londres et New York, Routledge, 1993, pp. 179-199.

Sinclair, $T$, ALIZADEH, P, and E.A.A ONUNGA, the Structure of Intemational Tourism and Tourism development in Kenya, in D. Harrison (ed.). Tourlsm and the Less Devoloped Countries. Londres, Bethaven Press, New York, Halsted Press, 1992, pp. 47.63.

Smith, V., Introduction, in V.H. Smith [ed.]. Hosts and Guests: the Anthropology of Tourism. Second Edition, Philadelphia, University of Pennsylvania Press, 1989, pp. 1-17.

Tourism Authority Of Thailand (TAT), Annual Report Bangkok, 1983

Tourism Authority OF Thailand (TAT), Thalland Tourism Statistical Report 1990. Bangkok, 1991.

Tourism Authority OF Thailand (TAT). Statistique du tourisme en Thailande par province 1990 (en thail, Bangkok, non daté.

Wood. A.E., ethnic Tourism, the State, and Cunural Change in Southeast Asia, Annals of Tourism Research, vol. 11, 1984, pp. 353-374. 